\title{
Serotonin Transporters in Dopamine Transporter Imaging: A Head-to-Head Comparison of Dopamine Transporter SPECT Radioligands ${ }^{123}$ I-FP-CIT and ${ }^{123}$ I-PE2I
}

\author{
Morten Ziebell ${ }^{1}$, Signe Holm-Hansen ${ }^{1}$, Gerda Thomsen ${ }^{1}$, Aase Wagner ${ }^{2}$, Peter Jensen ${ }^{1}$, Lars H. Pinborg ${ }^{1,3}$, \\ and Gitte Moos Knudsen ${ }^{1}$ \\ ${ }^{1}$ Neurobiology Research Unit and Cimbi, Rigshospitalet and University of Copenhagen, Copenhagen, Denmark; ${ }^{2}$ Diagnostic \\ Radiology, Rigshospitalet and University of Copenhagen, Copenhagen, Denmark; and ${ }^{3}$ Department of Neurology, Epilepsy Clinic, \\ Rigshospitalet and University of Copenhagen, Copenhagen, Denmark
}

Current SPECT radioligands available for in vivo imaging of the dopamine transporter (DAT) also show affinity for monoamine transporters other than DAT, especially the serotonin transporter (SERT). The effect of this lack of selectivity for in vivo imaging is unknown. In this study, we compared the SPECT radioligands $\quad{ }^{123} \mid-2-\beta$-carbomethoxy-3 $\beta$-(4-iodophenyl)- $N$-(3fluoropropyl)nortropane (123/-FP-CIT) and ${ }^{123} \mathrm{I}-\mathrm{N}$-(3-iodoprop$2 \mathrm{E}$-enyl)-2- $\beta$-carbomethoxy-3 $\beta$-(4-methylphenyl) ( $\left.{ }^{123} \mathrm{I}-\mathrm{PE} 2 \mathrm{I}\right)$. ${ }^{123} \mathrm{I}-\mathrm{FP}-\mathrm{CIT}$ has a 10 -fold higher selectivity than ${ }^{123} \mathrm{I}$-FP-CIT for DAT versus SERT. Methods: Sixteen healthy individuals were scanned in random order with both radioligands. The radioligands were administered according to standard recommendations: ${ }^{123}$ I-FP-CIT was given as a bolus injection, and the ratio between the striatum and reference tissue was measured after $3 \mathrm{~h}$. ${ }^{123}$-PE2I was administered in a bolus-infusion setup, and the nondisplaceable binding potential $\left(\mathrm{BP}_{\mathrm{ND}}\right)$ was measured after $2 \mathrm{~h}$. To assess the contribution of SERT to the overall SPECT signal, SERT was blocked by intravenous citalopram in 6 of the individuals. Results: The striatum-to-reference ratio -1 of 123 I-FP-CIT was on average $18 \%$ higher than the striatal $\mathrm{BP}_{\mathrm{ND}}$ of ${ }^{123}$-PE2I. Equal doses of radioactivity resulted in 3 times higher counting rates for ${ }^{123} \mid-F P-C I T$ than for ${ }^{123} \mid-P E 2 I$, both in target and in reference brain regions. Citalopram infusion led to significant reductions in both striatal $(22.8 \% \pm$ $20.4 \%, P<0.05)$ and thalamic $(63.0 \% \pm 47.9 \%, P<0.05)$ ${ }^{123}$ I-FP-CIT binding ratios, whereas $\mathrm{BP}_{\mathrm{ND}}$ of ${ }^{123}$ I-PE2I was unaltered. Likewise, blocking of SERT led to increased $(21 \% \pm$ $30.1 \%, P<0.001)$ plasma ${ }^{123}$ I-FP-CIT, probably as a result of significant blocking of peripheral SERT binding sites. By contrast, plasma ${ }^{123}$ I-PE2I remained stable. Conclusion: ${ }^{123}$ I-FP-CIT and ${ }^{123}$ I-PE2I had approximately the same targetto-background ratios, but per injected megabecquerel, ${ }^{123}$ I-FP$\mathrm{CIT}$ gave rise to 3 -fold higher cerebral counting rates. We found that ${ }^{123}$ I-FP-CIT, but not ${ }^{123} \mathrm{I}-\mathrm{PE} 2 \mathrm{I}$, brain images have a highly interindividual but significant signal contribution from SERT. Whether the SERT signal contribution is of clinical importance needs to be established in future patient studies.

Received Apr. 19, 2010; revision accepted Sep. 7, 2010.

For correspondence contact: Gitte Moos Knudsen, Neurobiology Research Unit, Rigshospitalet, N9201, 9 Blegdamsvej, Copenhagen, DK-2100 Denmark. E-mail: gmk@nru.dk

COPYRIGHT @ 2010 by the Society of Nuclear Medicine, Inc.

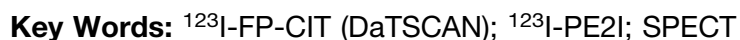

J Nucl Med 2010; 51:1885-1891

DOI: 10.2967/jnumed.110.078337

C ocaine labeled with ${ }^{11} \mathrm{C}$ was the first radioligand to be used for imaging the cerebral dopamine transporter (DAT) in vivo (1), but several other radioligands have subsequently been developed and tested as PET or SPECT ligands. With the introduction of $2-\beta$-carbomethoxy-3 $\beta$-(4-iodophenyl)tropane ( ${ }^{123} \mathrm{I}-\beta$-CIT) in 1991 (2), brain imaging of the DAT became a clinically useful method for supplementary diagnosis of movement disorders. A couple of serious drawbacks with ${ }^{123} \mathrm{I}-\beta$-CIT are that the radioligand has equal affinity for DATs and serotonin transporters (SERT) and that the slow kinetics of the ligand require imaging to be conducted $24 \mathrm{~h}$ after bolus injection.

2 - $\beta$-carbomethoxy-3 $\beta$-(4-iodophenyl)- $N$-(3-fluoropropyl)nortropane (FP-CIT) was synthesized in 1994 (3); its cerebral distribution and dosimetry in healthy volunteers was subsequently reported by Booij et al. in 1998 (4). ${ }^{123}$ I-FPCIT, compared with ${ }^{123}$ I- $\beta$-CIT, has an improved selectivity for DAT versus SERT (Table 1), and because of its lower DAT affinity ${ }^{123}$ I-FP-CIT has much faster kinetic properties, with a striatal peak time at 148 min after intravenous injection. Faster kinetic properties mean that with ${ }^{123}$ I-FP-CIT, the time between tracer injection and SPECT could be reduced to $3 \mathrm{~h}$, making it more feasible for use in the scanning of outpatients (5). With a bolus injection and without measurements of the arterial input function, the outcome parameter (usually the binding ratio between a target and a reference region) does, however, become sensitive to intersubject variation in the plasma clearance rate of the radioligand (6). ${ }^{123} \mathrm{I}-\mathrm{FP}-\mathrm{CIT}$ was licensed as DaTSCAN (Amersham Health) in Europe in 2000, and since then it has become a frequently used SPECT radioligand, particularly as an ancillary tool to diagnose patients with movement disorders. In 2006, more than 500 European SPECT 
centers were using ${ }^{123}$ I-FP-CIT for clinical purposes (7). The radioligand has also been widely used in both preclinical and clinical studies; a PubMed search in January 2010 shows that ${ }^{123}$ I-FP-CIT currently is mentioned in over 275 scientific papers.

${ }^{123}$ I-labeled $N$-(3-iodoprop-2E-enyl)-2- $\beta$-carbomethoxy$3 \beta$-(4-methylphenyl), named PE2I (MAP Medical Technologies), was synthesized in 1997 (8), and a dosimetry study in humans was published in 1998 (9). The ligand has about a 30-fold higher affinity for DAT than for SERT (Table 1), and because of its lower affinity to DAT ${ }^{123}$ I-PE2I has faster kinetics than ${ }^{123} \mathrm{I}-\mathrm{FP}-\mathrm{CIT}$, with a striatal peak time between 30 and 60 min. Quantification of ${ }^{123}$ I-PE2I binding to DAT is possible using kinetic or graphical analysis after bolus injection of the tracer and SPECT for 90 min or as a combination of bolus and constant infusion, for which unvarying levels in plasma and brain tissue are achieved after approximately $2 \mathrm{~h}(10,11)$. PE2I has also proven suitable as an ${ }^{11} \mathrm{C}$-labeled PET probe (12). In ${ }^{123} \mathrm{I}$-labeled form, neither PE2I nor FP-CIT gives rise to radiolabeled metabolites that can permeate the blood-brain barrier $(13,14)$. Despite its favorable properties, ${ }^{123} \mathrm{I}-\mathrm{PE} 2 \mathrm{I}$ is currently not licensed as a SPECT radioligand for clinical use.

The aim of this study was to compare ${ }^{123} \mathrm{I}-\mathrm{PE} 2 \mathrm{I}$ and ${ }^{123} \mathrm{I}-$ FP-CIT in a head-to-head design in terms of their binding parameters in various brain regions of interest (ROIs) and examine the significance of the SERT contribution to the total signal. The contribution was addressed by blocking the SERT with acute citalopram (Seropram; Lundbeck A/S) infusion.

\section{MATERIALS AND METHODS}

Sixteen healthy individuals (mean age $\pm \mathrm{SD}, 43.1 \pm 16.7 \mathrm{y}$; age range, 21-69 y; 10 men) were included; a diagram of the study design is shown in Figure 1. All subjects gave written informed consent. The study was performed in accordance with the ethical standards of the Declaration of Helsinki and was approved by the ethical committee of Copenhagen Capital Region (protocol H-B2008-024).

The healthy volunteers were recruited through advertising on the Internet or in newspapers. None of the subjects had any history of neurologic or psychiatric disorders, and they all claimed no drug or alcohol abuse. None smoked, nor were any taking medication. Cerebral MRI scans were obtained before the first

TABLE 1

SERT and DAT Binding Affinity of Inhibitor $\left(\mathrm{K}_{\mathrm{i}}\right)$, as Measured in Rat Brain Homogenates

\begin{tabular}{|c|c|c|c|}
\hline Ligand & $\begin{array}{c}\text { Ki DAT }(n M), \\
{ }^{3} \mathrm{H}-\mathrm{GBR}-12935\end{array}$ & $\begin{array}{l}\mathrm{K}_{\mathrm{i}} \mathrm{SERT}(\mathrm{nM}) \\
{ }^{3} \mathrm{H} \text {-paroxetine }\end{array}$ & $\begin{array}{l}\text { SERT-to-DAT } \\
\text { ratio }\end{array}$ \\
\hline${ }^{123} \mathrm{I}-\beta-\mathrm{CIT}^{*}$ & 27 & 3 & 0.1 \\
\hline${ }^{123} \mathrm{I}-\mathrm{FP}-\mathrm{CIT}^{\dagger}$ & 3.5 & 9.7 & 2.8 \\
\hline 123I-PE2| * & 17 & 500 & 29.4 \\
\hline \multicolumn{4}{|c|}{$\begin{array}{l}{ }^{*} \text { According to study of Abi-Dargham et al. (5). } \\
{ }^{\dagger} \text { According to study of Emond et al. (8). }\end{array}$} \\
\hline
\end{tabular}

SPECT scan, to exclude structural abnormalities and signs of vascular pathology. The results of routine urine drug tests and routine blood tests were normal in all subjects.

\section{Experimental Procedures}

Radioligand Administration. Cannulas were inserted into both cubital veins for radioligand administration and blood sampling. To block thyroidal uptake of free radioiodine, all subjects received $200 \mathrm{mg}$ of potassium perchloride intravenously $30 \mathrm{~min}$ before either ${ }^{123}$ I-PE2I or ${ }^{123}$ I-FP-CIT injection.

Baseline Experiments. In 10 subjects, the radioligands were administered according to standardized protocols, adapted to the differences in the kinetic properties $(5,10)$.

${ }^{123}$ I-PE2I. A bolus-infusion protocol of 2.7 (i.e., the bolus is worth $2.7 \mathrm{~h}$ of infusion) was used $(10,11)$. An average intravenous bolus of $2.0 \pm 0.1 \mathrm{~mL}$ (mean, 77.1 MBq [range, 65.3-91.7 MBq]) of ${ }^{123}$ I-PE2I was administered. The intravenous bolus was immediately followed by a constant infusion (for a total of $3 \mathrm{~h}$ ) of $3 \pm$ $0.1 \mathrm{~mL}$ (mean, 103.7 MBq [range, 92.3-124.2 MBq]) of ${ }^{123}$ I-PE2I dissolved in $43 \mathrm{~mL}$ of saline. The mean injected mass of PE2I was $1 \times 10^{-3} \mu \mathrm{g} / \mathrm{mL}$. Six SPECT acquisitions, each lasting $10 \mathrm{~min}$, were obtained between 120 and $180 \mathrm{~min}$ after ${ }^{123}$ I-PE2I injection.

${ }^{123} \mathrm{I}-\mathrm{FP}$-CIT. An average intravenous bolus of $2.0 \pm 0.1 \mathrm{~mL}$ (mean, 190.2 MBq [range, 184.3-202.2 MBq]) of ${ }^{123}$ I-FP-CIT was administered. The mean injected mass of FP-CIT was 0.1 $\mu \mathrm{g} / \mathrm{mL}$. Six SPECT acquisitions, each lasting $10 \mathrm{~min}$, were obtained between 180 and 240 min after ${ }^{123}$ I-FP-CIT injection.

There was an average of $10 \mathrm{~d}$ (range, 7 to $14 \mathrm{~d}$ ) between the ${ }^{123}$ I-FP-CIT and ${ }^{123}$ I-PE2I scan; scans were acquired in randomized order.

Citalopram Experiments. Six additional subjects participated in a citalopram experiment. Citalopram $(40 \mathrm{mg} / \mathrm{mL})$ was given at a dose of $0.15 \mathrm{mg} / \mathrm{kg}$ of body weight. The infusion lasted $30 \mathrm{~min}$ and was administered using an infusion pump.

For ${ }^{123}$ I-FP-CIT, the subjects underwent 2 SPECT scannings (1 d apart): a baseline scan and second scan that was immediately preceded by citalopram injection before the SPECT acquisition. For both scans, $24 \times 10$-min acquisitions (complete time-activity curves) were obtained between 0 and $240 \mathrm{~min}$.

For ${ }^{123}$ I-PE2I, the subjects underwent only 1 SPECT scanning. The acquisition was extended to $1 \mathrm{~h}$, with a maintained bolus-toinfusion ratio. One hundred minutes after ${ }^{123}$ I-PE2I injection, a 40-min baseline scan was obtained, and then citalopram infusion was started and scanning data were acquired for an additional $100 \mathrm{~min}$. Frames from 100 to 140 and from 140 to $240 \mathrm{~min}(8 \times$ 5 and $10 \times 10 \mathrm{~min}$, respectively) were obtained.

All subjects were monitored with standard electrocardiogram and blood pressure measurements.

Blood Sampling. Venous blood was collected every $10 \mathrm{~min}$ at the SPECT frame mid time. The sample was centrifuged, plasma was collected, and octanol was added to derive the lipophilic phase containing the radioligand parent compound (14). The sample was shaken and centrifuged, the octanol phase was pipetted into a counting vial, and, finally, radioactivity was measured in a $\gamma$-counter (Cobra II; Packard Instrument Co.).

Citalopram concentrations in blood were measured at 30 and $90 \mathrm{~min}$ after citalopram injection.

SPECT Acquisition and Image Reconstruction. SPECT images were acquired with a triple-head IRIX camera (Philips Medical) fitted with low-energy, general all-purpose, parallel-hole collimators (spatial resolution, $8.5 \mathrm{~mm}$ at $10 \mathrm{~cm}$ ). The mean radius of 


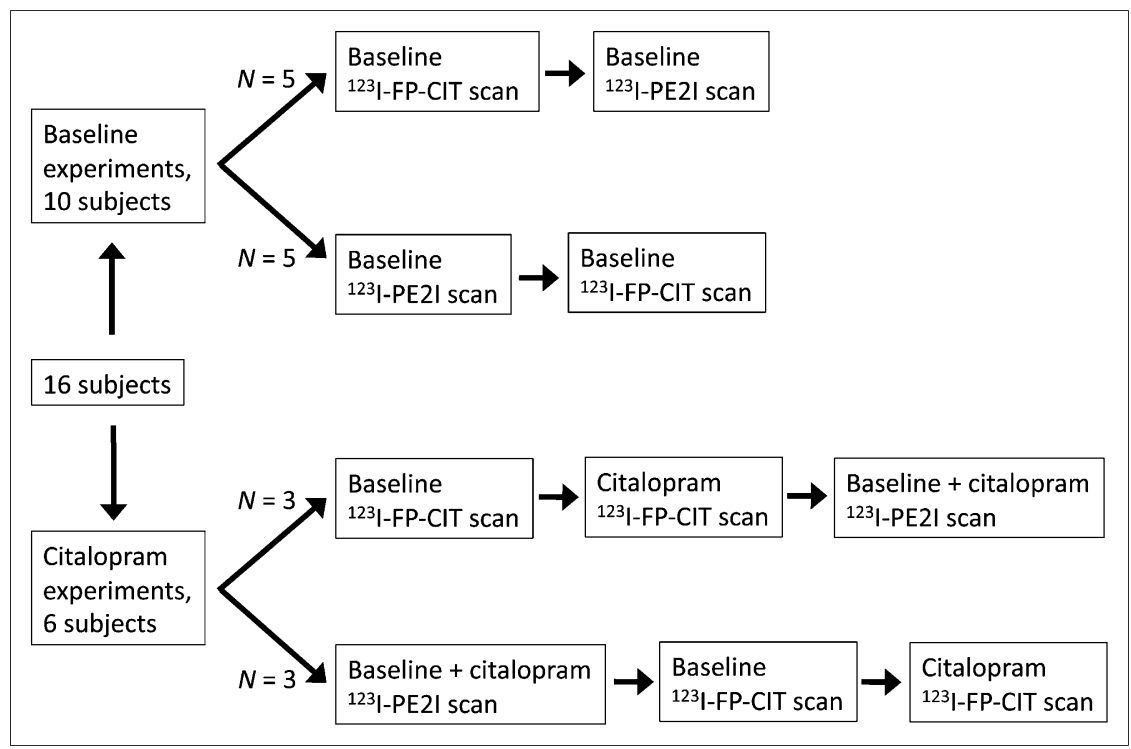

FIGURE 1. Flowchart of study design. Ten subjects were randomized to receive either ${ }^{123}|-P E 2|$ or ${ }^{123}$-FP-CIT for first scan. Six additional subjects were randomized to receive either ${ }^{123}$ |-PE2I or ${ }^{123 \mid-F P-C I T ~ f o r ~}$ baseline scan before corresponding citalopram challenge scan. To use all baseline experiments for head-to-head comparison of ${ }^{123}$ |-PE2I or ${ }^{123}$ |-FP-CIT, we also included baseline (i.e., without drug) experiments from citalopram experiments, so that total number of individuals with baseline and no-drug experiments was 16.

rotation was $15.2 \mathrm{~cm}$. Each head covered $120^{\circ}$ of the circular orbit. Scans were obtained and stored at fixed angles, with an angular interval of $3^{\circ}$.

The images were reconstructed with a MATLAB 6.5 (The MathWorks)-based program in $128 \times 128$ matrices $(2.33-\mathrm{mm}$ pixels and identical slice thickness) using standard filtered backprojection with a low-pass fourth-order Butterworth filter at 0.3 Nyquist $\left(0.64 \mathrm{~cm}^{-1}\right)$. This method is discussed in further detail in the study by Ziebell et al. (15).

\section{ROIs}

ROIs were manually delineated because we previously have shown that manually derived delineation is comparable to MRIguided ROI delineation in DAT SPECT quantification (15). This comparability is probably because these small ROIs can move easily out of the hot spot, despite the use of external fiducial markers, which should improve coregistration accuracy. Therefore, delineation of ROIs in DAT SPECT images is only a close estimate but never a $100 \%$ exact anatomic ROI with regard to the size, shape, and positioning, no matter the method used (manual delineation directly on the SPECT image, population-based template-guided delineation, delineation based on coregistration with another brain image that provides structural information, such as MRI) (15).

The ROIs were outlined by 1 delineator with more than $5 \mathrm{y}$ of experience; in the case of the ${ }^{123}$ I-FP-CIT scans, the delineator was unaware of whether citalopram had been administered. ROIs were delineated directly on the reconstructed SPECT images, guided by the use of Kretschmann and Weinrich's neuroanatomic atlas (16). Direct adaptation of ROI coordinates from the atlas required the use of a MATLAB program (The MathWorks) adapted in-house to reorient the reconstructed images to the canthomeatally oriented plane. ROIs were delineated on 5 consecutive slices $(2.3 \mathrm{~mm} / \mathrm{slice})$ in a summed image from all SPECT frames acquired from the scanning.

The ROIs were first randomly delineated either on the ${ }^{123} \mathrm{I}$-FPCIT image or on the ${ }^{123}$ I-PE2I image. Then the 2 images were coregistered using visual assessment, and the ROI was transferred to the image of the other radioligand. Finally, the placement of the
ROIs was adjusted to get the maximum counts per ROI, without changing the size or shape of the ROI. ROIs were optimized to eliminate coregistration errors.

\section{Derivation of Binding Parameters}

Because ${ }^{123} \mathrm{I}$-FP-CIT is administered as a bolus alone and ${ }^{123} \mathrm{I}-$ PE2I as a bolus followed by a constant infusion, binding parameters are not directly comparable in the baseline experiments $(n=$ 10). In these experiments, ${ }^{123}$ I-FP-CIT was measured from 3 to $4 \mathrm{~h}$ after bolus injection, and ${ }^{123}$ I-FP-CIT was therefore not in a true steady-state condition. With ${ }^{123}$ I-PE2I, however, specific binding to nondisplaceable binding is measured at steady-state conditions, which are reached between 100 and $180 \mathrm{~min}$ (10).

It is only at true steady-state conditions that the ratio of specific to nondisplaceable binding is equal to the binding potential of the nondisplaceable radioligand $\left(\mathrm{BP}_{\mathrm{ND}}\right)(17)$. Therefore, in the ${ }^{123} \mathrm{I}-$ FP-CIT baseline experiments we refer to the outcome as binding ratios and not $\mathrm{BP}_{\mathrm{ND}}$.

$\mathrm{BP}_{\mathrm{ND}}$ was calculated as:

$$
\mathrm{BP}_{\mathrm{ND}}=\frac{\text { Total radioligand }}{\text { Nondisplaceable radioligand }}-1,
$$

where nondisplaceable equals the sum of the free and nonspecific bound radioligand concentration represented by the cerebellum, which is assumed to be devoid of DAT.

To do an exact comparison of $\mathrm{BP}_{\mathrm{ND}}$ between the 2 radioligands in the citalopram experiments $(n=6)$, we had to calculate true ${ }^{123}$ I-FP-CIT $\mathrm{BP}_{\mathrm{ND}}$, which is done by obtaining full ${ }^{123}$ I-FP-CIT time-activity curves and applying simplified reference tissue modeling (SRTM) (18). ${ }^{123}$ I-PE2I is in true a steady-state condition; thus, we could conduct a within-scan pharmacologic SERT displacement with citalopram while continuing to acquire the time-activity curves. SRTM was performed with PMOD software (version 3.0; PMOD Technologies).

Changes in the plasma concentrations of the radioligand after citalopram administration were calculated differently for the 2 radiotracers. For ${ }^{123}$ I-FP-CIT, we used the plasma time-activity data to calculate the area under the curve, and for ${ }^{123}$ I-PE2I we 
took advantage of ${ }^{123}$ I-PE2I attaining tracer steady state and simply compared plasma concentration before, during, and after the pharmacologic challenge.

\section{Statistical Analysis}

All statistical analyses were performed with GraphPad Prism (version 5.00; GraphPad Software Inc.). Unless otherwise stated, all values are given as mean $\pm \mathrm{SD}$.

A 2-tailed paired $t$ test was used to compare binding ratios and $\mathrm{BP}_{\mathrm{ND}}$ values obtained with the 2 radioligands. $P$ values below 0.05 were considered statistically significant. All values are given with 95\% confidence interval. Because of the small sample size in the citalopram experiments $(n=6)$, we used a nonparametric Wilcoxon matched-pairs signed rank sum test.

\section{RESULTS}

\section{Counting Rates and Binding}

Compared with ${ }^{123} \mathrm{I}-\mathrm{PE} 2 \mathrm{I}$, for ${ }^{123} \mathrm{I}$-FP-CIT the average counting rates were $3.0 \pm 0.9$ (range, 1.7-4.8) times higher in the striatum, $3.4 \pm 0.9$ (range, 2.1-5.6) times higher in the thalamus, and $2.7 \pm 0.6$ (range, 1.7-4.0) times higher in the cerebellum. The individual striatal- and thalamic-to-cerebellum binding ratios or $\mathrm{BP}_{\mathrm{ND}} \mathrm{S}$ are shown in Figure 2. The average binding ratio was $3.6 \pm 0.7$ for ${ }^{123} \mathrm{I}$-FP-CIT, which was significantly higher than the $\mathrm{BP}_{\mathrm{ND}}$ of $3.0 \pm 0.5$ for ${ }^{123}$ I-PE2I ( $t$ test, $P<0.005$ ). One individual had an ${ }^{123} \mathrm{I}-$ FP-CIT binding ratio lower than the ${ }^{123} \mathrm{I}-\mathrm{PE} 2 \mathrm{I} \mathrm{BP}_{\mathrm{ND}}$; the remaining 15 individuals had between $0 \%$ and $30 \%$ higher ${ }^{123}$ I-FP-CIT binding ratios. The average thalamus-to-cerebellum binding ratio was $0.9 \pm 0.3$ for ${ }^{123} \mathrm{I}-\mathrm{FP}-\mathrm{CIT}-$ significantly higher than the $\mathrm{BP}_{\mathrm{ND}}$ of $0.5 \pm 0.2$ for ${ }^{123} \mathrm{I}-\mathrm{PE} 2 \mathrm{I}$ ( $t$ test, $P<0.001$ ). Seen consistently in all individuals, the individual thalamus binding ratio was between $10 \%$ and $800 \%$ higher for ${ }^{123} \mathrm{I}-\mathrm{FP}-\mathrm{CIT}$ than the $\mathrm{BP}_{\mathrm{ND}}$ was for ${ }^{123} \mathrm{I}-$ PE2I. Because the thalamus has 20 times more SERT than DAT binding sites and the proportions are opposite in the striatum (19-21), we calculated the thalamic-to-striatal ratio as proxy for in vivo DAT selectivity (Fig. 3). This ratio was $46 \%$ higher for ${ }^{123}$ I-FP-CIT than for ${ }^{123}$ I-PE2I

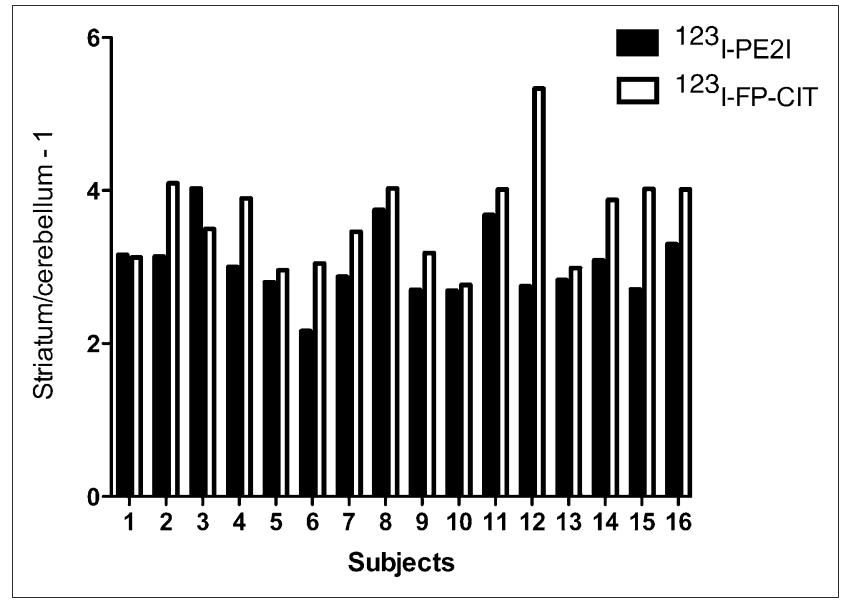

FIGURE 2. Individual ratios of specific to nonspecific striatal binding $(n=16)$. ${ }^{123}$-FP-CIT binding ratio is significantly higher than ${ }^{123}$ I-PE2I BP ${ }_{\text {ND }}(t$ test, $P<0.05)$.

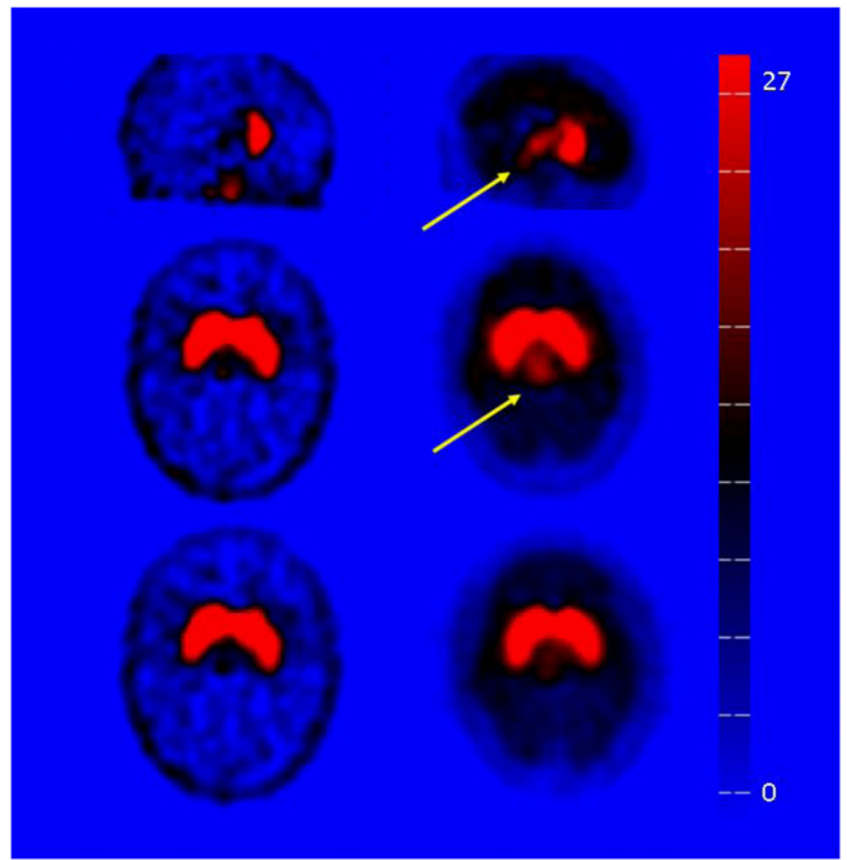

FIGURE 3. ${ }^{123}|-P E 2|$ image (left) and ${ }^{123}$-FP-CIT image (right) of same individual. In first 2 rows (sagittal and horizontal slices), thalamus is clearly visualized in ${ }^{123}$ I-FP-CIT image (arrows). Bottom row shows images after acute SERT blocking with citalopram.

( $t$ test, $P<0.005$ ). For both radioligands, the ratio of caudate to putamen binding was not significantly different from 1 ( $t$ test, $P>0.1)$. Finally, the standard reference regions were compared; there was no statistically significant difference for either radioligand between mean counts in occipital cortex and cerebellum ( $t$ test, $P>0.5$ ).

\section{Citalopram Experiments}

All subjects, except for 1, received the planned dose of $0.15 \mathrm{mg}$ of citalopram per kilogram of body weight. One volunteer experienced nausea and vomiting $15 \mathrm{~min}$ after citalopram administration was initiated; therefore, the infusion was stopped (at the current dose of $0.11 \mathrm{mg} / \mathrm{kg}$ ) and symptoms disappeared within a few minutes. For the second scan, $0.11 \mathrm{mg}$ of citalopram per kilogram was administered over $30 \mathrm{~min}$ (as in the first scan), and this time the subject did not experience any side effects. One subject had a mild drop in blood pressure, from 110 to $95 \mathrm{~mm} \mathrm{Hg}$, lasting for $2 \mathrm{~h}$. The other 5 volunteers felt slightly lightheaded for a few minutes toward the end of the infusion, but this effect disappeared a few minutes after citalopram infusion was terminated. The mean plasma citalopram concentration at 30 and $90 \mathrm{~min}$ after radiotracer injection was $52.2 \pm 11.4$ and $47.3 \pm 9.6 \mathrm{nmol} / \mathrm{L}$, respectively. No difference in mean plasma citalopram concentrations between the 2 radioligands was observed: ${ }^{123} \mathrm{I}-\mathrm{FP}-\mathrm{CIT}, 47.0$ $\pm 10.0 \mathrm{nmol} / \mathrm{L}$, and ${ }^{123} \mathrm{I}-\mathrm{PE} 2 \mathrm{I}, 52.4 \pm 11.0 \mathrm{nmol} / \mathrm{L}$. After administration of citalopram, compared with the unblocked condition, the area under the curve of the venous blood samples for ${ }^{123}$ I-FP-CIT (from 0 to $240 \mathrm{~min}$ ) increased by $21.2 \%$ (range, $1.6 \%-82.0 \%$ ) (Wilcoxon, $P<0.05$ ). Вy 
contrast, citalopram infusion did not alter ${ }^{123}$ I-PE2I plasma radioactivity. Acute citalopram treatment resulted in an average decrease in ${ }^{123} \mathrm{I}$-FP-CIT $\mathrm{BP}_{\mathrm{ND}}$ values of $22.8 \%$ (range, $-9.0 \%-43.2 \%$ ) in the striatum (Wilcoxon, $P<$ 0.05 ) and of $63.0 \%$ (range, $19.0 \%-131.3 \%$ ) in the thalamus (Wilcoxon, $P<0.05$ ). When the ratio method (based on 180-240 min) was used instead, these numbers were $15.6 \%$ (range, $0.5 \%-38.0 \%$ ) and $86.3 \%$ (range, 25.2\%-125.7\%). There was no correlation between the relative decrease in $\mathrm{BP}_{\mathrm{ND}}$ in the striatum and thalamus within the single individual.

For ${ }^{123} \mathrm{I}-\mathrm{PE} 2 \mathrm{I}$, citalopram infusion did not alter $\mathrm{BP}_{\mathrm{ND}}$ in the striatum or thalamus (Wilcoxon, $P>0.1$ ). Figure 4 shows ${ }^{123}$ I-PE2I and ${ }^{123}$ I-FP-CIT images retrieved for the same individual; binding in the thalamus is easily appreciated in the ${ }^{123}$ I-FP-CIT image, disappearing after citalopram infusion. The individual $\mathrm{BP}_{\mathrm{ND}}$ in relation to the citalopram infusion is shown in Figures 5A and 5B. Figure $5 \mathrm{C}$ illustrates how the individual striatal $\mathrm{BP}_{\mathrm{ND}}$ obtained from ${ }^{123}$ I-FP-CIT approximates the $\mathrm{BP}_{\mathrm{ND}}$ obtained with ${ }^{123}$ I-PE2I after citalopram infusion. For ${ }^{123}$ I-FP-CIT, the SRTM fitted the time-activity curve from 0 to $240 \mathrm{~min}$. We estimated the distribution volume in the cerebellumthat is, the ratio of the total concentration of radioligand in the cerebellum to the total concentration of radioligand in plasma, using the venous blood samples from 0 to $240 \mathrm{~min}$. This distribution volume was $1.5 \pm 0.6 \mathrm{~Bq} / \mathrm{mL}$ (brain)/Bq/ $\mathrm{mL}$ (blood) for ${ }^{123}$ I-PE2I and $5.5 \pm 2.0$ for ${ }^{123}$ I-FP-CIT. In all 6 individuals, the SRTM-determined $\mathrm{BP}_{\mathrm{ND}}$ obtained with ${ }^{123}$ I-FP-CIT at baseline was significantly higher than $\mathrm{BP}_{\mathrm{ND}}$ for ${ }^{123} \mathrm{I}$-PE2I: ${ }^{123} \mathrm{I}$-FP-CIT, $4.2 \pm 0.7$, and ${ }^{123} \mathrm{I}$-PE2I, $3.1 \pm 0.4$ (Wilcoxon, $P<0.05$ ). This difference was, however, highly variable among the individuals, ranging from $5 \%$ to $93 \%$. The difference between ${ }^{123} \mathrm{I}$-PE2I $\mathrm{BP}_{\mathrm{ND}}$ and

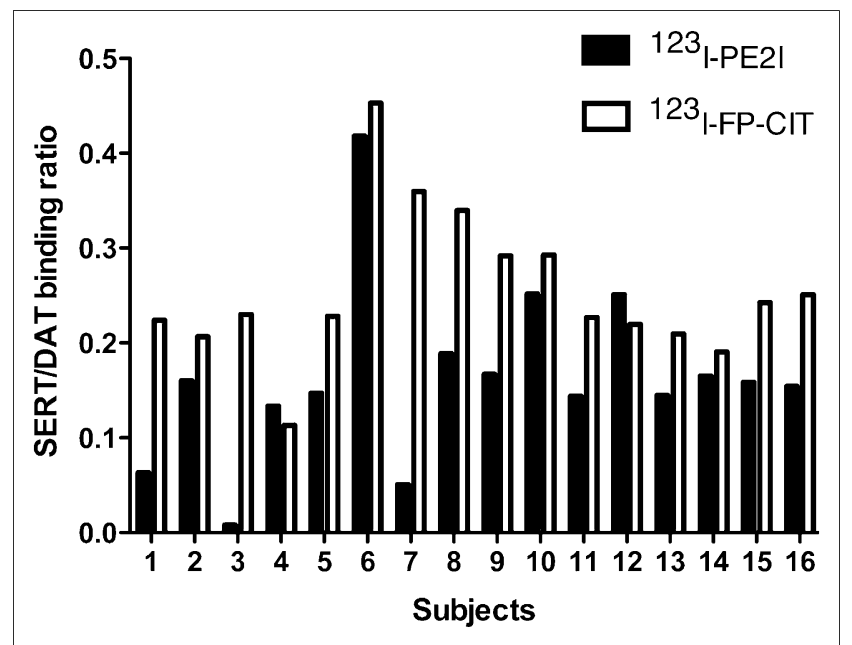

FIGURE 4. As proxy for in vivo SERT and DAT selectivity of ${ }^{123} \mid-$ PE2I and ${ }^{123}$-FP-CIT, individual specific-to-nonspecific ratio in thalamus to individual specific-to-nonspecific ratio in striatum is shown. Ratio for ${ }^{123}$ |-FP-CIT is significantly higher than for ${ }^{123}$ |PE2I ( $t$ test, $P<0.005, n=16$ ).

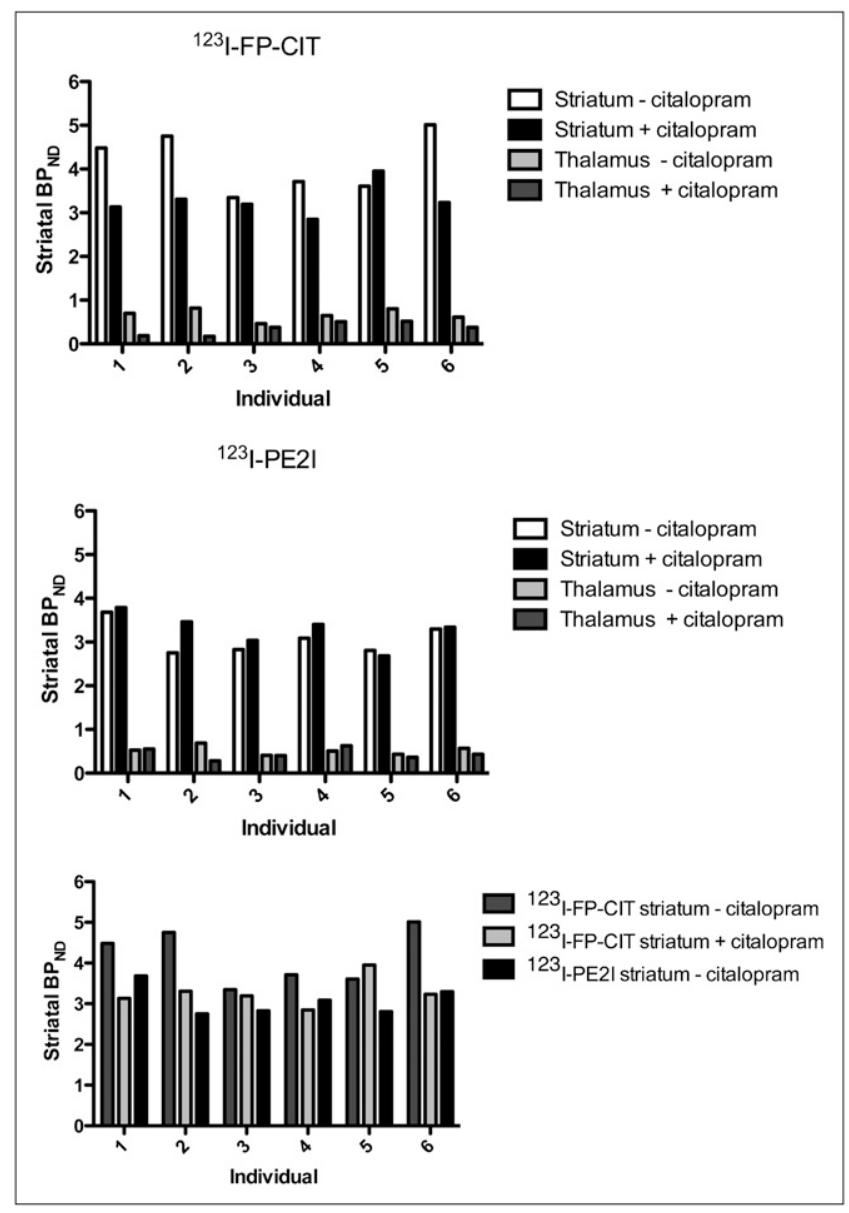

FIGURE 5. Response to citalopram infusion, expressed as change in $\mathrm{BP}_{\mathrm{ND}}$ in striatum and thalamus. $\mathrm{BP}_{\mathrm{ND}}$ for ${ }^{123} \mathrm{I}-\mathrm{FP}-\mathrm{CIT}$ was calculated with SRTM; $\mathrm{BP}_{\mathrm{ND}}$ for ${ }^{123}$ I-PE2I was calculated as ratio between steady-state tissue levels. (C) $\mathrm{BP}_{\mathrm{ND}}$ for ${ }^{123}$-FP-CIT approaches $\mathrm{BP}_{\mathrm{ND}}$ for ${ }^{123}$-PE2I after SERT blocking. Same subject was used for all experiments.

${ }^{123}$ I-FP-CIT binding ratio disappeared after citalopram infusion: ${ }^{123}$ I-FP-CIT, $3.3 \pm 0.4$, and ${ }^{123}$ I-PE2I, $3.3 \pm 0.4$ (Wilcoxon, $P>0.2$ ).

There was no significant difference in $\mathrm{BP}_{\mathrm{ND}}$ and simple binding ratio obtained from 180 to $240 \mathrm{~min}$ after ${ }^{123} \mathrm{I}-$ FP-CIT injection (Wilcoxon, $P>0.5$ ). The individual terminal venous plasma clearance was stable; on average, it decreased 5.9\% per hour in the fourth hour after bolus injection.

\section{DISCUSSION}

To our knowledge, this is the first study of a head-to-head design to compare DAT and SERT binding properties of the 2 SPECT DAT radioligands ${ }^{123}$ I-PE2I and ${ }^{123}$ I-FP-CIT and to assess the contribution of SERT to the total signal. In this study, we found 3 -fold higher brain counting rates per injected megabecquerel for ${ }^{123}$ I-FP-CIT than for ${ }^{123} \mathrm{I}-$ PE2I. The ratio of striatal specific to nondisplaceable binding for ${ }^{123}$ I-FP-CIT was almost $20 \%$ higher than the $\mathrm{BP}_{\mathrm{ND}}$ of ${ }^{123}$ I-PE2I, but after acute blocking of SERT with citalo- 
pram no significant difference in the specific-to-nondisplaceable striatal binding of ${ }^{123}$ I-FP-CIT versus ${ }^{123}$ I-PE2I was observed. Although ${ }^{123}$ I-PE2I BP ${ }_{\mathrm{ND}}$ was unaffected by citalopram infusion, ${ }^{123}$ I-FP-CIT $\mathrm{BP}_{\mathrm{ND}}$ declined significantly. Citalopram infusion increased the plasma concentration of ${ }^{123}$ I-FP-CIT but not of ${ }^{123}$ I-PE2I; this increase supported the finding that peripheral SERT binding sites, particularly in the lung tissue (4), were blocked, resulting in a higher availability of ${ }^{123}$ I-FP-CIT but not of ${ }^{123}$ I-PE2I.

Count statistics per injected megabecquerel for ${ }^{123}$ I-FPCIT are superior to those for ${ }^{123}$ I-PE2I; these better count statistics are particularly beneficial in patients with low DAT binding. In addition, the higher brain uptake of ${ }^{123} \mathrm{I}-$ FP-CIT is beneficial and, compared with ${ }^{123}$ I-PE2I, its higher uptake can be translated into a better determination of the target-to-background ratio or into a lower dose of radioactivity by shortening the scanning time.

We chose to compare the 2 radioligands using experimental setups most commonly used in the clinical settingthat is, bolus ( ${ }^{123}$ I-FP-CIT) and bolus-infusion ( ${ }^{123}$ I-PE2I) schedules. At the time of measurement, ${ }^{123}$ I-FP-CIT was not in a steady-state condition in plasma or in tissue but was in a transient equilibrium. In theory, the ${ }^{123}$ I-FP-CIT binding ratio overestimates the steady-state $\mathrm{BP}_{\mathrm{ND}}$ because of the effect of the terminal plasma clearance rate-a problem originally addressed by Carson et al. in 1993 (6). We found, however, that the actual terminal plasma clearance rate was stable, with an average of an approximately $6 \%$ decline per hour; second, there was no difference between $\mathrm{BP}_{\mathrm{ND}}$ calculated using SRTM and the binding ratio obtained as a mean from 180 to $240 \mathrm{~min}$ after ${ }^{123} \mathrm{I}$-FPCIT injection. Thus, transient equilibrium analysis of ${ }^{123} \mathrm{I}-$ FP-CIT does not explain the higher ratio for ${ }^{123}$ I-FP-CIT than for ${ }^{123}$ I-PE2I in healthy individuals. As a note of caution, however, altered plasma clearance may occur in patients with, for example, renal diseases or with altered metabolism of ${ }^{123}$ I-FP-CIT.

We also evaluated the potential confounding effect of SERT on the total striatal signal in what is presumed to be cerebral DAT imaging. Because of the following reasons, our results confirmed in vitro data, suggesting that the higher in vivo binding ratio of nondisplaceable radioligand for ${ }^{123} \mathrm{I}-$ FP-CIT than for ${ }^{123}$ I-PE2I was related to SERT binding. First, a significantly higher binding was seen in a SERT high-density region, the thalamus, for ${ }^{123} \mathrm{I}$-FP-CIT than for ${ }^{123}$ I-PE2I, both in absolute terms and when assessed as the thalamus-to-striatum ratio. Second, acute blockade of SERT before ${ }^{123}$ I-FP-CIT administration was associated with significantly increased plasma ${ }^{123}$ I-FP-CIT levels, because of the lower peripheral binding of ${ }^{123}$ I-FP-CIT. This increased plasma was not seen with ${ }^{123}$ I-PE2I. Finally, a significant decrease in $\mathrm{BP}_{\mathrm{ND}}$ (average, 24\%) of ${ }^{123}$ I-FP-CIT was observed in the striatum after SERT blocking, whereas no significant change in $\mathrm{BP}_{\mathrm{ND}}$ for ${ }^{123} \mathrm{I}-\mathrm{PE} 2 \mathrm{I}$ was observed.

We found a considerable interindividual variability in ${ }^{123}$ I-FP-CIT binding, both in comparison to ${ }^{123}$ I-PE2I bind- ing and in response to SERT blockade, with striatal $\mathrm{BP}_{\mathrm{ND}}$ decreases of between $-8 \%$ and $43 \%$ for ${ }^{123}$ I-FP-CIT. This variability could be because of the SERT density (density of transporters available to radioligand binding $\left[\mathrm{B}_{\text {avail }}\right]$ ), or affinity $\left(K_{d}\right)$ of the SERT for ${ }^{123}$ I-FP-CIT, $K_{d}$ of citalopram, or relative DAT versus SERT density ratio in that region. We considered the $K_{d}$ of citalopram a less likely reason because we did not observe any correlation between the effect of citalopram on $\mathrm{BP}_{\mathrm{ND}}$ for SERT low- and high-density regions.

In theory, if the observed higher striatal target-to-background ratio for ${ }^{123}$ I-FP-CIT than for ${ }^{123}$ I-PE2I should be explained by variations in the relative striatal densities of DAT and SERT, and assuming published in vitro $\mathrm{K}_{\mathrm{i}}$ values for the radioligands, then the striatal $\mathrm{B}_{\text {avail, DAT }}-$ to $-\mathrm{B}_{\text {avail, }}$ SERT ratio would need to be below 5:1 (Supplemental Appendix; supplemental materials are available online only at http://jnm.snmjournals.org). Human postmortem brain studies point toward a $\mathrm{B}_{\text {avail, DAT }}$-to- $\mathrm{B}_{\text {avail, SERT }}$ ratio of $20: 1$ in the putamen $(\sim 200: 10 \mathrm{pmol} / \mathrm{g})(20,21)$. This ratio could probably vary somewhat in healthy subjects, and a large variation in $\mathrm{BP}_{\mathrm{ND}}$ has indeed been reported in in vivo studies (SD of $\mathrm{BP}_{\mathrm{ND}}$ for SERT, 23\% of mean $(22,23)$, and for DAT, $13 \%$ of mean $(11,24)$ ). Hence, our large decrease in ${ }^{123}$ I-FP-CIT signal after citalopram blocking suggests that the superiority of ${ }^{123}$ I-PE2I over ${ }^{123}$ I-FP-CIT is even more selective in vivo than in vitro.

Acute blocking of the SERT decreased striatal $\mathrm{BP}_{\mathrm{ND}}$ as measured with ${ }^{123}$ I-FP-CIT, to the same level as that for ${ }^{123} \mathrm{I}$-PE2I. On the basis of the available in vitro data on ${ }^{123} \mathrm{I}-$ FP-CIT DAT versus SERT selectivity and affinity, the extent to which SERT blocking decreased striatal $\mathrm{BP}_{\mathrm{ND}}$ was larger than expected (Supplemental Appendix). Three previous studies have investigated the striatal ${ }^{123}$ I-FP-CIT binding in response to semichronic selective serotonin reuptake inhibitor treatment (25-27), and despite reporting significant binding to SERT sites in the lungs, increasedand not decreased-striatal binding ratios were reported. The main difference between these studies and our study is that selective serotonin reuptake inhibitor treatment was not administered acutely or intravenously, but over days and orally. It is possible that the more chronic administration induced alterations in both DAT and SERT, preventing a straightforward interpretation of the data.

Finally, we want to address the fact that $\mathrm{BP}_{\mathrm{ND}}$ is proportional to the free fraction of the nondisplaceable radioligand in the brain $\left(\mathrm{f}_{\mathrm{ND}}\right)$. It is hard to imagine that infusion of citalopram should occupy the total amount of protein binding sites in the brain affecting the $\mathrm{f}_{\mathrm{ND}}$ and thereby the nondisplaceable binding of ${ }^{123}$ I-FP-CIT. Further, if such an effect would occur, it would increase the $f_{\mathrm{ND}}$ and not decrease it, leading to an increase in ${ }^{123} \mathrm{I}-\mathrm{FP}-\mathrm{CIT} \mathrm{BP}_{\mathrm{ND}}$.

\section{CONCLUSION}

In the clinic, ${ }^{123}$ I-FP-CIT has the advantage of the experimental simplicity of a bolus-alone setup, and as we have shown here, this radioligand has a substantially higher brain 
uptake than ${ }^{123}$ I-PE2I. The interval between ${ }^{123}$ I-FP-CIT injection and scanning is $3 \mathrm{~h}$, compared with $2 \mathrm{~h}$ for ${ }^{123} \mathrm{I}-$ PE2I, but patients scanned with ${ }^{123}$ I-PE2I are connected to an infusion pump and therefore restricted in their physical activities during these hours. The bolus-infusion setup is easily implemented for routine use. In our SPECT laboratory, we conduct more than 100 diagnostic ${ }^{123}$ I-PE2I SPECT scans annually. The number of acquisitions is the same for both radiotracers.

Yet, the simplicity of ${ }^{123}$ I-FP-CIT administration may compromise exact quantification of the binding. The mode of administration of ${ }^{123}$ I-FP-CIT makes it susceptible to interindividual variation in the terminal plasma clearance rate of the tracer, which may be a particular problem in medicated patients or in patients with concomitant medical disorders. However, for the healthy unmedicated subjects studied here, the influence of the terminal plasma clearance rate on the outcome parameter is not quantitatively important. The variation in terminal plasma clearance rate is, in theory, eliminated using the bolus-infusion approach we used for ${ }^{123}$ I-PE2I.

As we have shown in this study, the most important disadvantage of ${ }^{123}$ I-FP-CIT, compared with ${ }^{123}$ I-PE2I, is the lack of selectivity for DAT. This lack of selectivity is quantitatively important, as we demonstrated after citalopram blocking or displacement in healthy volunteers. The outcome parameter of a ${ }^{123} \mathrm{I}$-FP-CIT SPECT experiment is clearly more susceptible to up- and downregulation of striatal SERT and to coadministration of SERT blocking or modulating pharmaceuticals. The inferior DAT selectivity of ${ }^{123}$ I-FP-CIT, compared with ${ }^{123}$ I-PE2I, is likely to be more pronounced in patients with low DAT density in the striatum, as demonstrated in the Supplemental Appendix. This inferior selectivity has to be examined in similar studies of patients with low DAT density.

\section{ACKNOWLEDGMENTS}

We thank Svitlana Olsen and Glenna Skouboe for expert technical assistance and Kirsten Møller, PhD, DMSc, Jesper Poulsen, and Ronni Plovsing for precautionary measures during citalopram infusion. This work was supported by Rigshospitalet, the Lundbeck Foundation, the EC-FP6-project DiMI LSHB-CT-2005-512146, the Toyota Foundation, the University of Copenhagen, and HR (the Capital Region of Denmark). Part of the data were acquired in conjunction with the establishment of a European database of ${ }^{123}$ I-FP-CIT SPET scans of healthy controls (ENC-DAT) sponsored by the EANM/European Network of Excellence for Brain Imaging.

\section{REFERENCES}

1. Fowler JS, Volkow ND, Wolf AP, et al. Mapping cocaine binding sites in human and baboon brain in vivo. Synapse. 1989;4:371-377.

2. Neumeyer JL, Wang SY, Milius RA, et al. [ $\left.{ }^{123} \mathrm{I}\right]-2 \beta$-carbomethoxy-3 $\beta$-(4iodophenyl)tropane: high-affinity SPECT radiotracer of monoamine reuptake sites in brain. J Med Chem. 1991;34:3144-3146.

3. Neumeyer JL, Wang S, Gao Y, et al. $N$ - $\omega$-fluoroalkyl analogs of (1R)-2 $\beta$-carbomethoxy-3 $\beta$-(4-iodophenyl)-tropane $(\beta-\mathrm{CIT})$ : radiotracers for positron emis- sion tomography and single photon emission computed tomography imaging of dopamine transporters. J Med Chem. 1994;37:1558-1561.

4. Booij J, Busemann Sokole E, Stabin MG, Janssen AG, de Bruin K, van Royen EA. Human biodistribution and dosimetry of [ $\left.{ }^{123} \mathrm{I}\right] \mathrm{FP}-\mathrm{CIT}$ : a potent radioligand for imaging of dopamine transporters. Eur J Nucl Med. 1998;25:24-30.

5. Abi-Dargham A, Gandelman MS, DeErausquin GA, et al. SPECT imaging of dopamine transporters in human brain with iodine-123-fluoroalkyl analogs of ß-CIT. J Nucl Med. 1996;37:1129-1133.

6. Carson RE, Channing MA, Blasberg RG, et al. Comparison of bolus and infusion methods for receptor quantitation: application to $\left[{ }^{18} \mathrm{~F}\right]$ cyclofoxy and positron emission tomography. J Cereb Blood Flow Metab. 1993;13:24-42.

7. Booij J, Kemp P. Dopamine transporter imaging with $\left[{ }^{123} \mathrm{I}\right] \mathrm{FP}-\mathrm{CIT}$ SPECT: potential effects of drugs. Eur J Nucl Med Mol Imaging. 2008;35:424-438.

8. Emond $\mathrm{P}$, Garreau L, Chalon S, et al. Synthesis and ligand binding of nortropane

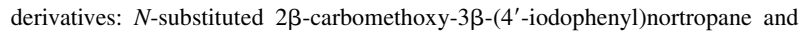

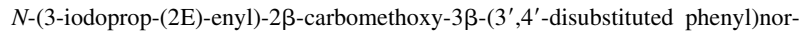
tropane-new high-affinity and selective compounds for the dopamine transporter. J Med Chem. 1997;40:1366-1372.

9. Kuikka JT, Baulieu JL, Hiltunen J, et al. Pharmacokinetics and dosimetry of iodine-123 labelled PE2I in humans, a radioligand for dopamine transporter imaging. Eur J Nucl Med. 1998;25:531-534.

10. Pinborg LH, Ziebell M, Frokjaer VG, et al. Quantification of ${ }^{123}$ I-PE2I binding to dopamine transporter with SPECT after bolus and bolus/infusion. J Nucl Med. 2005;46:1119-1127.

11. Ziebell M, Thomsen G, Knudsen GM, et al. Reproducibility of $\left[{ }^{123} \mathrm{I}\right] \mathrm{PE} 2 \mathrm{I}$ binding to dopamine transporters with SPECT. Eur J Nucl Med Mol Imaging. 2007;34:101-109.

12. Delorenzo C, Kumar JD, Zanderigo F, Mann JJ, Parsey RV. Modeling considerations for in vivo quantification of the dopamine transporter using $\left[{ }^{11} \mathrm{C}\right] \mathrm{PE} 2 \mathrm{I}$ and positron emission tomography. J Cereb Blood Flow Metab. 2009;29:1332-1345.

13. Seibyl JP, Marek K, Sheff K, et al. Iodine-123- $\beta$-CIT and iodine-123-FPCIT SPECT measurement of dopamine transporters in healthy subjects and Parkinson's patients. J Nucl Med. 1998;39:1500-1508.

14. Videbaek C. G.M. K, K. B. Octanol extraction yields similar results as HPLC for quantitation of [ [123I]PE2I metabolism [abstract]. Eur J Nucl Med. 1999;26:1139.

15. Ziebell M, Pinborg LH, Thomsen G, et al. MRI-guided region-of-interest delineation is comparable to manual delineation in dopamine transporter SPECT quantification in patients: a reproducibility study. J Nucl Med Technol. 2010;38:61-68.

16. Kretschmann H-J, Weinrich W. Neuroanatomy and Cranial Computed Tomography. Stuttgart, Germany: Thieme; 1986.

17. Innis RB, Cunningham VJ, Delforge J, et al. Consensus nomenclature for in vivo imaging of reversibly binding radioligands. J Cereb Blood Flow Metab. 2007; 27:1533-1539.

18. Lammertsma AA, Hume SP. Simplified reference tissue model for PET receptor studies. Neuroimage. 1996;4:153-158.

19. Hall H, Halldin C, Guilloteau D, et al. Visualization of the dopamine transporter in the human brain postmortem with the new selective ligand [ $\left.{ }^{125} \mathrm{I}\right] \mathrm{PE} 2 \mathrm{I}$. Neuroimage. 1999;9:108-116.

20. Madras BK, Gracz LM, Fahey MA, et al. Altropane, a SPECT or PET imaging probe for dopamine neurons: III. Human dopamine transporter in postmortem normal and Parkinson's diseased brain. Synapse. 1998;29:116-127.

21. Varnas K, Halldin C, Hall H. Autoradiographic distribution of serotonin transporters and receptor subtypes in human brain. Hum Brain Mapp. 2004;22:246-260.

22. Matsumoto R, Ichise $M$, Ito $H$, et al. Reduced serotonin transporter binding in the insular cortex in patients with obsessive-compulsive disorder: a $\left[{ }^{11} \mathrm{C}\right] \mathrm{DASB}$ PET study. Neuroimage. 2009;49:121-126.

23. Frankle WG, Slifstein M, Gunn RN, et al. Estimation of serotonin transporter parameters with ${ }^{11} \mathrm{C}$-DASB in healthy humans: reproducibility and comparison of methods. J Nucl Med. 2006;47:815-826.

24. Booij J, Habraken JB, Bergmans P, et al. Imaging of dopamine transporters with iodine-123-FP-CIT SPECT in healthy controls and patients with Parkinson's disease. J Nucl Med. 1998;39:1879-1884.

25. Booij J, de Jong J, de Bruin K, Knol R, de Win MM, van Eck-Smit BL. Quantification of striatal dopamine transporters with ${ }^{123}$ I-FP-CIT SPECT is influenced by the selective serotonin reuptake inhibitor paroxetine: a double-blind, placebocontrolled, crossover study in healthy control subjects. J Nucl Med. 2007;48: 359-366.

26. de Win MM, Habraken JB, Reneman L, van den Brink W, den Heeten GJ, Booij J. Validation of $\left[{ }^{123} \mathrm{I}\right] \beta$-CIT SPECT to assess serotonin transporters in vivo in humans: a double-blind, placebo-controlled, crossover study with the selective serotonin reuptake inhibitor citalopram. Neuropsychopharmacology. 2005;30:996-1005.

27. Kugaya A, Seneca NM, Snyder PJ, et al. Changes in human in vivo serotonin and dopamine transporter availabilities during chronic antidepressant administration. Neuropsychopharmacology. 2003;28:413-420. 